\title{
Damage regularisation with inertia gradients
}

\author{
Terry Bennett ${ }^{\mathrm{a}, *}$, Antonio Rodríguez-Ferran ${ }^{\mathrm{b}}$, Harm Askes $^{\mathrm{a}}$ \\ ${ }^{a}$ Department of Civil and Structural Engineering, University of Sheffield, Sir Frederick Mappin Building Mappin Street, Sheffield S1 3JD, United Kingdom \\ ${ }^{b}$ Laboratori de Càlcul Numèric (LaCàN), Departament de Matemàtica Aplicada III, Universitat Politècnica de Catalunya, Barcelona, Spain
}

A B S T R A C T

A gradient enhanced model is formulated which simulates micro structurally induced wave dispersion and is capable of achieving mesh objective results when modelling strain softening materials. Length scale parameters are introduced to incorporate both micro inertial effects and strain smoothing. The model is formulated such that all the gradient enhancement terms are contained within the kinetic energy functional, whilst all non linear terms are linked to the strain energy functional. In addition, discretisation of the governing equations is performed in a manner such that only $\mathscr{C}^{0}$ continuity is required and is symmetric in the elastic range. Dispersion analysis is performed to show the correct format that a constitutive model must conform to in order that regularisation of strain softening material models will occur. This dispersion analysis is then used to explore how the two length scales may influence the width of the zone in which strain localisation takes place. The efficacy of the model in simulating strain softening behaviour in a mesh objective manner and the effects of wave dispersion on damage initiation and accumulation are demonstrated in numerical examples.

\section{Introduction}

Achieving mesh objective simulations with numerical solution procedures has long been recognised as being of vital importance in the modelling of strain softening materials in statics and dynamics. Proposed solutions for regularisation of strain localisation such as the crack band model (Bažant and Oh, 1983) serve to regularise the energy dissipation, however strain localisation still occurs within the width of single element or width attributable to an integration point, hence mesh dependent responses result from calculations. To alleviate this problem integral non locality (Pijaudier Cabot and Bažant, 1987), gradient non local techniques (Peerlings et al., 1996; Muhlhaus and Aifantis, 1991) and visco plasticity (Sluys, 1992) all serve as a device to supplement the constitutive relationship with an internal length scale. This length scale then limits the width of the zone in which localisation of strains is permitted. A similar internal length scale is introduced into the static modelling of solids in the field of Gradient Elasticity. The aim being to model effectively micro scale effects in heterogeneous materials or the removal of singularities that may occur in classical elasticity, for example at crack tips and dislocations (Ru and Aifantis, 1993; Gutkin and Aifantis, 1999). A micro structural length scale serves the added

\footnotetext{
* Corresponding author. Fax: +44114222 5700 .

E-mail address: t.bennett@sheffield.ac.uk (T. Bennett).
}

purpose of naturally incorporating size effects into a model (Aifantis, 1999). In dynamics, gradient elasticity has been used to great effect to incorporate wave dispersion phenomena. This phenomenon is induced from material heterogeneity as the wave length of a given signal approaches the physical dimensions of a problem. The use of a single, inertia related, length scale has been used to capture this phenomenon effectively (Chen and Fish, 2001; Wang and Sun, 2002). The combination of both micro structural and micro inertial length scales can be traced back to the pio neering work of Mindlin (1964) with simplified versions of this model being advocated more recently (Metrikine and Askes, 2002; Georgiadis, 2003). A frequently encountered problem when incor porating higher order derivatives into a numerical model is the more stringent continuity requirements of the numerical dis cretisation. The Ru-Aifantis theorem (Ru and Aifantis, 1993) may be invoked to circumvent this problem by transforming the fourth order equilibrium equations into a coupled set of equations con taining only second order terms, resulting in only $\mathscr{C}^{0}$ continuity requirements as used in the standard implementation of the finite element method. The model presented is formulated such that all non local effects can be contained within the kinetic energy potential. This provides a clear separation of the non local and non linear effects into the kinetic potential and strain energy potential, respectively. This separation carries over to the finite element implementation: the non local effects are only present in the mass matrix and do not interfere with the constitutive routines that 
affect the stiffness matrix. As a result, the implementation is generic and can be coupled to a wide range of dissipative material laws. The remainder of the paper is organised as follows. The gradient elasticity 'backbone' model is formulated in Section 2 with an outline of the damage mechanics model in Section 2.1. In Section 2.2 an analysis of dispersive waves which investigates the correct format that the constitutive model must adopt, and in addition provides the critical wave length, i.e. the width of the damaging zone, is performed. With the format of the constitutive model now determined, the governing equations are extended to allow for non linearities and subsequently discretised for numerical analyses in Section 2.3. Numerical examples of the model efficacy in achieving mesh objective simulations in one and two dimensions are performed in Section 3, followed by an analysis of the effects of dispersion upon damage initiation and accumulation. The key findings are then elucidated in the concluding remarks.

\section{Model formulation}

A 'backbone' dynamic gradient elasticity model is introduced (Askes et al., 2007; Bennett et al., 2007). The equations of motion for classical elasto dynamics are simultaneously extended with strain gradients and inertia gradients as postulated by Mindlin (1964). Mindlin also adopted a simplification of the inertia gradi ents that was suggested earlier by Toupin (1962); furthermore, a simplification of the strain gradients follows from the later work of Aifantis (1992), so that in its simplest form the model with strain gradients and inertia gradients, cast in terms of macroscopic displacements $\mathbf{u}^{\mathrm{M}}$ can be written as

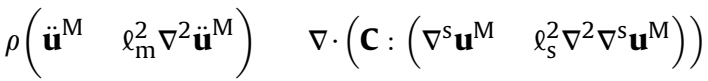

where $\mathbf{C}$ is the fourth order tensor containing the elastic moduli, $\ell_{S}$ is an internal length scale related to higher order stiffness, $\mathbf{u}_{\mathrm{g}}$ and $\mathbf{u}_{\mathrm{g}}$ are the gradient enriched displacement and acceleration fields, respectively (the subscript $g$ is used to denote gradient enrichment), $\rho$ is the mass density and $\ell_{\mathrm{m}}$ is a second length scale related to higher order inertia. ${ }^{1}$ Realistic simulations of wave dispersion can be obtained under the condition $\ell_{\mathrm{m}}>\ell_{\mathrm{s}}$ (Metrikine and Askes, 2002, 2006; Askes and Metrikine, 2002; Askes et al., 2007). The model of Equation (1) has also been used in applications of wave propagation by Georgiadis and coworkers (Georgiadis et al., 2000; Georgiadis, 2003). Invoking the Ru-Aifantis theorem (Ru and Aifantis, 1993) and defining an auxiliary microscopic displacement field

$$
\mathbf{u}^{\mathrm{m}} \quad \mathbf{u}^{\mathrm{M}} \quad \ell_{\mathrm{m}}^{2} \nabla^{2} \mathbf{u}^{\mathrm{M}}
$$

after some algebraic manipulations, as described by Askes et al. (2007), we can arrive at a set of coupled equations

$\left.\rho \frac{\ell_{\mathrm{m}}^{2}}{l_{\mathrm{s}}^{2}} \ddot{\mathbf{u}}^{\mathrm{m}} \quad \frac{\ell_{\mathrm{m}}^{2} \quad \ell_{\mathrm{s}}^{2}}{l_{\mathrm{s}}^{2}} \ddot{\mathbf{u}}^{\mathrm{M}}\right) \quad \nabla \cdot\left(\mathbf{C}: \nabla^{\mathrm{s}} \mathbf{u}^{\mathrm{m}}\right)$

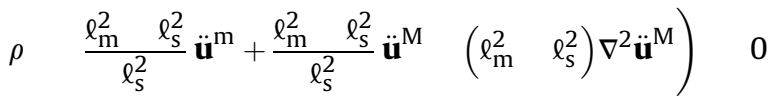

that replaces equation (1). Throughout, the superscripts $\mathrm{m}$ and $\mathrm{M}$ indicate microscopic and macroscopic displacements, respectively.

\footnotetext{
${ }^{1}$ It has been argued that the two length scales $\ell_{\mathrm{s}}$ and $\ell_{\mathrm{m}}$ can be related to the sizes of the Representative Volume Elements in statics and dynamics, respectively, Gitman et al. (2005). However, at present it is unclear whether this would also be possible in a non-linear context such as that adopted in here, and this issue would require further study.
}

The formulation remains symmetrical in the elastic range and contains only second order spatial derivative terms, allowing the system to be solved using only $\mathscr{C}^{0}$ continuity in discretised form. Identifying the kinematic and potential energy functionals, deno ted by superscripts kin and pot respectively, from equations (3) and (4) as

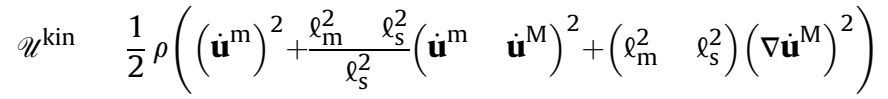

where the notations $(\dot{\mathbf{u}})^{2} \quad \dot{\mathbf{u}} \cdot \dot{\mathbf{u}}$ and $(\nabla \dot{\mathbf{u}})^{2} \quad \nabla \dot{\mathbf{u}}: \nabla \dot{\mathbf{u}}$ have been adopted, and

$$
\mathscr{U}^{\text {pot }} \quad \frac{1}{2} \varepsilon^{\mathrm{m}}: \mathbf{C}: \varepsilon^{\mathrm{m}}
$$

it is immediately apparent that all gradient enhanced terms are contained solely within the kinematic energy functional. Similarly the fourth order tensor of elastic moduli in the potential energy functional can be replaced by inelastic moduli, thus allowing a model formulation to be constructed with a clear separation between the non local and non linear terms.

\subsection{Constitutive relation}

For the purpose of this study attention is restricted to continuum damage mechanics, however the model is discretised in a manner such that incorporation of any existing constitutive model is straightforward and will benefit from the gradient enrichments in a natural manner without major changes to developed constitutive models. The constitutive relationship for isotropic damage is written as

$\boldsymbol{\sigma} \quad\left(1 \omega\left(\varepsilon^{b}\right)\right) \mathbf{C}: \varepsilon^{a}$

where $\omega$ is the damage variable which can take the value $\omega \quad 0$ for virgin material through to $\omega 1$ for fully degraded material. $\varepsilon^{a}$ and $\varepsilon^{b}$ are strain tensor measures, which if chosen to be the 'local' strains one can recover the standard, local, continuum damage mechanics model, well known to produce a mesh dependent response. The correct choice of these strain tensor measures is discussed further in the following dispersion analysis of Section 2.2. For clarity, we restrict our attention throughout this paper to a simple bilinear stress-strain diagram as shown in Fig. 1. In one dimension this is obtained through a damage loading function given by

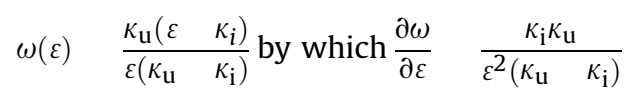

where $\kappa_{\mathrm{i}}$ is the damage initiation strain and $\kappa_{\mathrm{u}}$ is the ultimate admissible strain. In a multi dimensional setting, $\varepsilon$ in equation (8) is replaced with a scalar equivalent strain measure $\widehat{\varepsilon}$. In the two

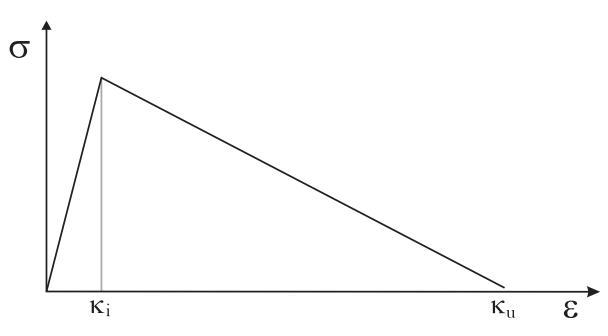

Fig. 1. Damage model. 
dimensional examples shown in Section 3.2 we choose the equiv alent strain, after Mazars (1986), as

$\widehat{\varepsilon} \sqrt{\sum_{i}^{2}\left\langle\varepsilon_{i}\right\rangle^{2}}$

where $\left\langle>\right.$ are the Macaulay brackets and $\varepsilon_{i}$ are the principal strains.

\subsection{Dispersion analysis}

An analysis of the wave dispersion characteristics of the current model is performed in one dimension with the aim of investigating the appropriate format of the constitutive equation and deter mining the influence of the length scales on the critical wave length, thus determining the width of the localisation zone. The one dimensional governing equations are written as

$\rho \ddot{\mathbf{u}}^{\mathrm{M}} \quad \rho \ell_{\mathrm{m}}^{2} \frac{\mathrm{d}^{2} \ddot{\mathbf{u}}^{\mathrm{M}}}{\mathrm{d} x^{2}} \quad \frac{\mathrm{d} \boldsymbol{\sigma}\left(\varepsilon^{\mathrm{m}}, \varepsilon^{\mathrm{M}}\right)}{\mathrm{d} x} \quad \frac{\partial \boldsymbol{\sigma}}{\partial \varepsilon^{\mathrm{m}}} \frac{\mathrm{d}^{2} \mathbf{u}^{\mathrm{m}}}{\mathrm{d} x^{2}}+\frac{\partial \boldsymbol{\sigma}}{\partial \varepsilon^{\mathrm{M}}} \frac{\mathrm{d}^{2} \mathbf{u}^{\mathrm{M}}}{\mathrm{d} x^{2}}$

Equation (10) is used together with the relation between the microscopic and macroscopic displacements

$\mathbf{u}^{\mathrm{m}} \quad \mathbf{u}^{\mathrm{M}} \quad \ell_{\mathrm{s}}^{2} \frac{\mathrm{d}^{2} \mathbf{u}^{\mathrm{M}}}{\mathrm{d} x^{2}}$

A uniform reference state of strain $\varepsilon_{0}$ is assumed, resulting from a displacement field $u_{0}$. Infinitesimal perturbations from the reference state of $\delta \boldsymbol{u}^{\mathrm{m}} A_{1} \exp \left(\mathrm{i} k\left(\begin{array}{ll}x & c t)\end{array}\right)\right.$ and $\delta \boldsymbol{u}^{\mathrm{M}} \quad A_{2} \exp (\mathrm{i} k(x \quad c t))$ are taken, where $k$ and $c$ are the wave number and the phase velocity, respectively, and the two ampli tudes are denoted $A_{1}$ and $A_{2}$. This yields a microscopic displace ment field $\boldsymbol{u}^{\mathrm{m}} u_{0}+\delta \boldsymbol{u}^{\mathrm{m}}$ together with a macroscopic field $\boldsymbol{u}^{\mathrm{M}} \quad u_{0}+\delta \boldsymbol{u}^{\mathrm{M}}$. A relation between the two amplitudes is found by substituting the two perturbations into equation (11), which yields

$A_{1} \quad A_{2}\left(1+k^{2} \ell_{\mathrm{s}}^{2}\right)$

Similarly, substituting the two perturbations into equation (10) renders

$\rho c^{2} A_{2}\left(1+k^{2} \ell_{\mathrm{m}}^{2}\right) \quad A_{1} \frac{\partial \sigma}{\partial \varepsilon^{\mathrm{m}}}+A_{2} \frac{\partial \sigma}{\partial \varepsilon^{\mathrm{M}}}$

Finally, substituting equation (12) into equation (13) gives

$\frac{c^{2}}{c_{\mathrm{e}}^{2}}\left(1+k^{2} \ell_{\mathrm{m}}^{2}\right) \quad \frac{1}{E}\left[\frac{\partial \sigma}{\partial \varepsilon^{\mathrm{m}}}\left(1+k^{2} \ell_{\mathrm{s}}^{2}\right)+\frac{\partial \sigma}{\partial \varepsilon^{\mathrm{M}}}\right]$

where $c_{\mathrm{e}} \quad \sqrt{ } E / \rho$ is the elastic bar velocity. Four different formats of the constitutive law in equation (7) could be envisaged, depending on which strain $\left(\varepsilon^{\mathrm{m}}\right.$ or $\left.\varepsilon^{\mathrm{M}}\right)$ is used. However, inspection of equation (3) shows that $\varepsilon^{a}$ must be taken as $\varepsilon^{\mathrm{m}}$ in this model. A dispersion analysis of all potential formats of the constitutive model is given by Rodríguez Ferran et al. (2011). The first format of

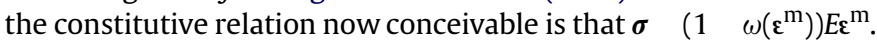
When this is substituted into equation (14), one obtains

$\frac{c^{2}}{c_{\mathrm{e}}^{2}}\left(1+k^{2} \ell_{\mathrm{m}}^{2}\right) \quad \frac{\kappa_{\mathrm{i}}}{\kappa_{\mathrm{u}} \quad \kappa_{\mathrm{i}}}\left(1+k^{2} \ell_{\mathrm{s}}^{2}\right)$

which leads to imaginary phase velocities $c$ for all wave numbers $k$ and is therefore not regularised (note that $\kappa_{\mathrm{u}}>\kappa_{\mathrm{i}}>0$ ). This result is unsurprising as this is identical to a 'local' damage model. Next, it is assumed that $\sigma \quad\left(1 \omega\left(\varepsilon^{\mathrm{M}}\right)\right) E \varepsilon^{\mathrm{m}}$. This is combined with equation (14) and yields

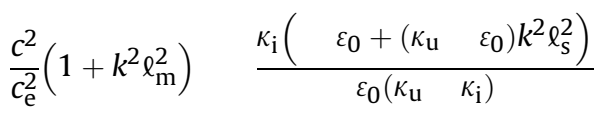

A range of wave numbers exist for which the phase velocities are real, thus this format is suitable for regularisation. The width of the zone in which strain localisation is active can be found by setting c 0 for the appropriate formats; the wave number for which $c \quad 0$ is termed the critical wave number $k_{\text {crit }}$. The associated wave length is denoted the critical wave length $\lambda_{\text {crit }}$. For the present formulation $\left(\varepsilon^{a} \quad \varepsilon^{\mathrm{m}}, \varepsilon^{b} \quad \varepsilon^{\mathrm{M}}\right)$ it is found that

$\lambda_{\text {crit }} \quad \frac{2 \pi}{k_{\text {crit }}} \quad 2 \pi \ell_{\mathrm{s}} / \frac{\kappa_{\mathrm{u}} \quad \varepsilon_{0}}{\varepsilon_{0}}$

Note that this expression only depends on the stiffness related length scale $\ell_{\mathrm{s}}$, not on the inertia related length scale $\ell_{\mathrm{m}}$.

\subsection{Numerical discretisation of governing equations}

The derivation of the weak form of equations (3) and (4) and its finite element discretisation is standard, and leads to

$\rho \frac{\ell_{\mathrm{m}}^{2}}{\ell_{\mathrm{s}}^{2}} \mathbf{M} \ddot{\mathbf{u}}^{\mathrm{m}} \quad \rho \frac{\ell_{\mathrm{m}}^{2} \ell_{\mathrm{s}}^{2}}{\ell_{\mathrm{s}}^{2}} \mathbf{M} \ddot{\mathbf{u}}^{\mathrm{M}}+\mathbf{f}^{\mathrm{int}}\left(\mathbf{u}^{\mathrm{m}}, \mathbf{u}^{\mathrm{M}}\right) \quad \mathbf{f}^{\mathrm{ext}}$

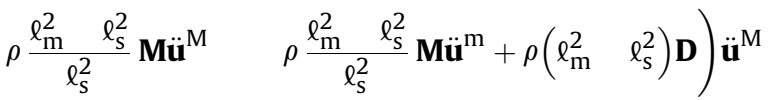

where $\mathbf{u}^{\mathrm{m}}$ and $\mathbf{u}^{\mathrm{M}}$ now represent the nodal displacements, similarly $\mathbf{u}^{\mathrm{m}}$ and $\mathbf{u}^{\mathrm{M}}$ now represent the nodal accelerations. $\mathbf{f}^{\mathrm{ext}}$ are the external forces on the system and the internal forces $\mathbf{f}^{\text {int }}$. The 'mass' and 'diffusivity' matrices, are defined as

M $\int_{\Omega} \mathbf{N}^{\mathrm{T}} \mathbf{N} \mathrm{d} \Omega$

and

D $\int_{\Omega} \nabla \mathbf{N}^{\mathrm{T}} \nabla \mathbf{N} \mathrm{d} \Omega$

with $\mathbf{N}$ the matrix of shape functions and $\nabla \mathbf{N}$ the matrix of shape function gradients. The Voigt notation is used, so $\sigma, \varepsilon^{\mathrm{m}}$ and $\varepsilon^{\mathrm{M}}$ now represent the usual finite element vectors rather than tensors. The internal force vector is defined as

$\mathbf{f}^{\mathrm{int}} \quad \int_{\Omega} \mathbf{B}^{\mathrm{T}} \boldsymbol{\sigma}\left(\varepsilon^{\mathrm{m}}, \varepsilon^{\mathrm{M}}\right) \mathrm{d} \Omega$

where $\mathbf{B}$ is the usual matrix of shape function derivatives. The integrals in equations (20) and (22) are typically approximated by means of a Gaussian quadrature.

\subsubsection{Linearisation}

We recast equations (18) and (19) as

$$
\begin{aligned}
\mathbf{r}_{\text {equil }}\left(\mathbf{u}^{\mathrm{m}}, \mathbf{u}^{\mathrm{M}}\right):= & \mathbf{f}^{\mathrm{int}}\left(\mathbf{u}^{\mathrm{m}}, \mathbf{u}^{\mathrm{M}}\right) \quad \mathbf{f}^{\text {ext }} \rho \frac{\ell_{\mathrm{m}}^{2}}{\ell_{\mathrm{s}}^{2}} \mathbf{M} \ddot{\mathbf{u}}^{\mathrm{M}} \\
& +\rho \frac{\ell_{\mathrm{m}}^{2} \ell_{\mathrm{s}}^{2}}{\ell_{\mathrm{s}}^{2}} \mathbf{M}^{\mathrm{m}} \quad 0
\end{aligned}
$$

and 


$$
\begin{aligned}
& \mathbf{r}_{\text {regu }}\left(\mathbf{u}^{\mathrm{m}}, \mathbf{u}^{\mathrm{M}}\right):=\rho \frac{\ell_{\mathrm{m}}^{2} \quad \ell_{\mathrm{s}}^{2}}{l_{\mathrm{s}}^{2}} \mathbf{M} \ddot{\mathbf{u}}^{\mathrm{M}} \quad \rho \frac{\ell_{\mathrm{m}}^{2} \quad \ell_{\mathrm{s}}^{2}}{\ell_{\mathrm{s}}^{2}} \mathbf{M}^{\mathrm{m}} \\
& \rho\left(\begin{array}{ll}
l_{\mathrm{m}}^{2} & \ell_{\mathrm{s}}^{2}
\end{array}\right) \mathbf{D} \ddot{\mathbf{u}}^{\mathrm{M}} \quad 0
\end{aligned}
$$

where $\mathbf{r}_{\text {equil }}$ is the non linear equilibrium residual and $\mathbf{r}_{\text {regu }}$ is the regularisation residual. Consistent linearisation of equations (23) and (24) is required for their solution, which results in

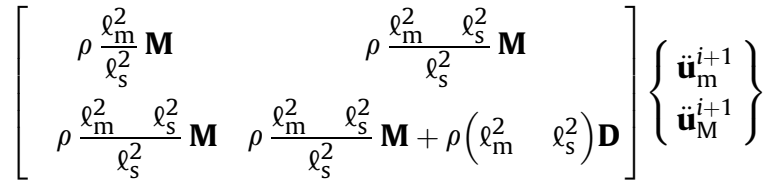

$$
\begin{aligned}
& +\left[\begin{array}{cc}
\mathbf{K}_{\mathrm{mm}}^{i} & \mathbf{K}_{\mathrm{mM}}^{i} \\
0 & 0
\end{array}\right]\left\{\begin{array}{l}
\delta \mathbf{u}_{\mathrm{m}}^{i} \\
\delta \mathbf{u}_{\mathrm{M}}^{i}
\end{array}\right\} \quad\left\{\begin{array}{c}
\mathbf{r}_{\text {equil }}^{i} \\
0
\end{array}\right\}
\end{aligned}
$$

where $\delta \mathbf{u}$ is the iterative correction in the displacements, $i$ is the iteration number, and the stiffness matrices are

$$
\begin{gathered}
\mathbf{K}^{\mathrm{mm}}:=\frac{\partial \mathbf{r}_{\text {equil }}}{\partial \mathbf{u}^{\mathrm{m}}} \quad \int_{\Omega} \mathbf{B}^{\mathrm{T}} \frac{\partial \boldsymbol{\sigma}\left(\varepsilon^{\mathrm{m}}, \varepsilon^{\mathrm{M}}\right)}{\partial \varepsilon^{\mathrm{m}}} \mathbf{B} \mathrm{d} \Omega \\
\mathbf{K}^{\mathrm{mM}}:=\frac{\partial \mathbf{r}_{\text {equil }}}{\partial \mathbf{u}^{\mathrm{M}}} \quad \int_{\Omega} \mathbf{B}^{\mathrm{T}} \frac{\partial \boldsymbol{\sigma}\left(\varepsilon^{\mathrm{m}}, \varepsilon^{\mathrm{M}}\right)}{\partial \varepsilon^{\mathrm{M}}} \mathbf{B} \mathrm{d} \Omega
\end{gathered}
$$

The damage model that yields mesh objective results, as shown in Section 2.2 , in a multi dimensional setting is

$$
\boldsymbol{\sigma} \quad\left(1 \omega\left(\varepsilon^{\mathrm{M}}\right)\right) \mathbf{C}: \varepsilon^{\mathrm{m}}
$$

The tangent operators for this model are

$\frac{\partial \boldsymbol{\sigma}\left(\varepsilon^{\mathrm{m}}, \varepsilon^{\mathrm{M}}\right)}{\partial \varepsilon^{\mathrm{m}}} \quad\left(1 \quad \omega\left(\varepsilon^{\mathrm{M}}\right)\right) \mathbf{C}$

and

$\frac{\partial \boldsymbol{\sigma}\left(\varepsilon^{\mathrm{m}}, \varepsilon^{\mathrm{M}}\right)}{\partial \varepsilon^{\mathrm{M}}} \quad \mathbf{C}_{\varepsilon}{ }^{\mathrm{m}} \frac{\partial \omega}{\partial \varepsilon^{\mathrm{m}}}$

thus the stiffness matrices (26) and (27) are the usual secant matrix

$\mathbf{K}^{\mathrm{mm}} \quad \int_{\Omega} \mathbf{B}^{\mathrm{T}}\left[\begin{array}{ll}1 & \left.\omega\left(\varepsilon^{\mathrm{M}}\right)\right]\end{array}\right] \mathbf{C B} \mathrm{d} \Omega$

and the tangent contribution

$$
\mathbf{K}^{\mathrm{mM}} \quad \int_{\Omega} \mathbf{B}^{\mathrm{T}} \mathbf{C}_{\varepsilon}^{\mathrm{m}} \frac{\partial \omega}{\partial \varepsilon^{\mathrm{M}}} \mathbf{B} \mathrm{d} \Omega
$$

The model in equation (25) is symmetric whilst the material behaviour is elastic; after damage initiates symmetry is lost due to the tangent contribution $\mathbf{K}^{\mathrm{mM}}$.

\section{Examples}

Three examples of the method are presented using benchmark tests adapted from Sluys (1992), with the goal of demonstrating that the proposed framework is simultaneously capable of regu larising strain softening and incorporating dispersion effects. In the first two examples, the efficacy of the model to regularise softening is of primary interest, thus for clarity, dispersion effects are ignored. In order to nullify the dispersion element of the model the micro

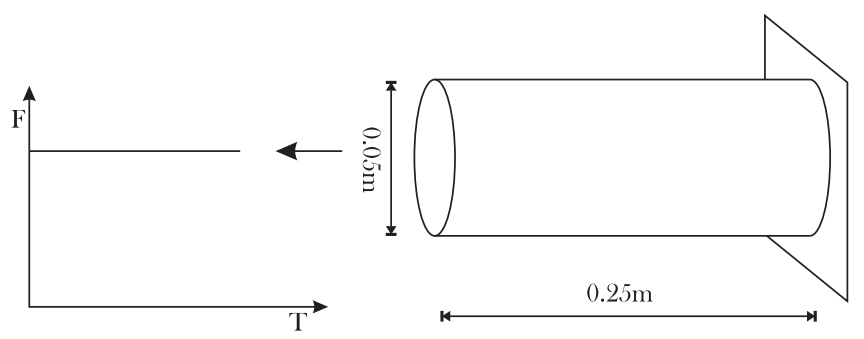

Fig. 2. One-dimensional bar.

inertia length scale is set equal to the stiffness length scale, $\ell_{m} \quad \ell_{s}$, see Askes et al. (2007). In the third example, the emphasis is upon the effects of dispersive behaviour on the damage process, thus different $\ell_{\mathrm{m}}$ values, or more pertinently different $\ell_{\mathrm{m}}$ to $l_{\mathrm{s}}$ ratios, are employed.

\subsection{One dimensional bar}

A $0.25 \mathrm{~m}$ long bar with a diameter of $0.05 \mathrm{~m}$ is subjected to a Heaviside forcing function of $3.0 \mathrm{kN}$ at its left hand face, see Fig. 2, is modelled in one dimension. The resulting tensile stress wave travels the length of the bar until it reflects at the fixed right hand end, returning back along the bar as a tensile wave with double the original magnitude. This doubled magnitude, now in excess of the material tensile strength, initiates damage within the material. The properties of the bar are chosen to be representative of concrete, with a Young's modulus $E \quad 30 \times 10^{9} \mathrm{~N} / \mathrm{m}^{2}$ and mass density $\rho 2000 \mathrm{~kg} / \mathrm{m}^{3}$, thus producing a wave speed of $c_{\mathrm{e}} \quad \sqrt{ } E / \rho \quad 3873 \mathrm{~m} / \mathrm{s}$. The damage is idealised as having a linear softening regime as shown in Fig. 1, with an initiation strain of $\kappa_{\mathrm{i}} \quad 0.8 \times 10^{-4}$ and ultimate strain $\kappa_{\mathrm{u}} \quad 1.25 \times 10^{-3}$. Time step size $\Delta t$ is chosen to be equal to $h / c_{\mathrm{e}}$, where $h$ is the element size, so as to reduce numerical dispersion as demonstrated by Bennett and Askes (2008). The total time of the simulations is set such that an elastic wave can propagate 1.5 times the total length of the bar, $t \quad(1.5 \times L) / c_{\mathrm{e}}(1.5 \times 0.25) / 387396.8 \mu \mathrm{s}$, plotted results are shown for this end of simulation time. Fig. 3 shows the effect of the micro structural length scale $\ell_{s}$ in controlling the width of the process zone, a constant element size of $0.5 \times 10^{-3} \mathrm{~m}$. By comparing the damage profiles for $\ell_{\mathrm{s}} 3 \times 10^{-3} \mathrm{~m}$ and $5 \times 10^{-3} \mathrm{~m}$, it can be seen that the micro structural length scale spreads the damage over a wider area, and for these simulations which have

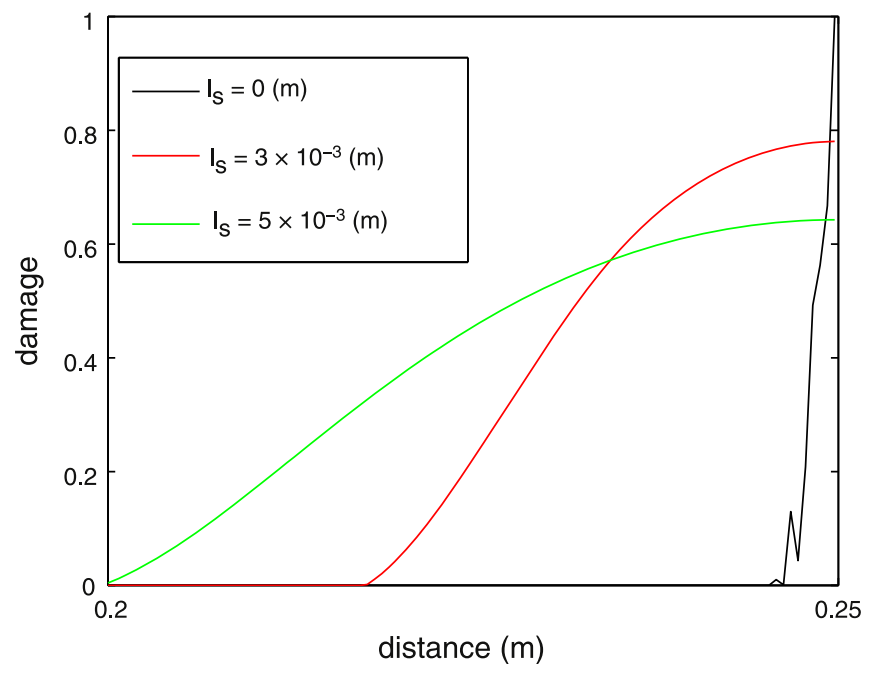

Fig. 3. Regularisation properties of model. 


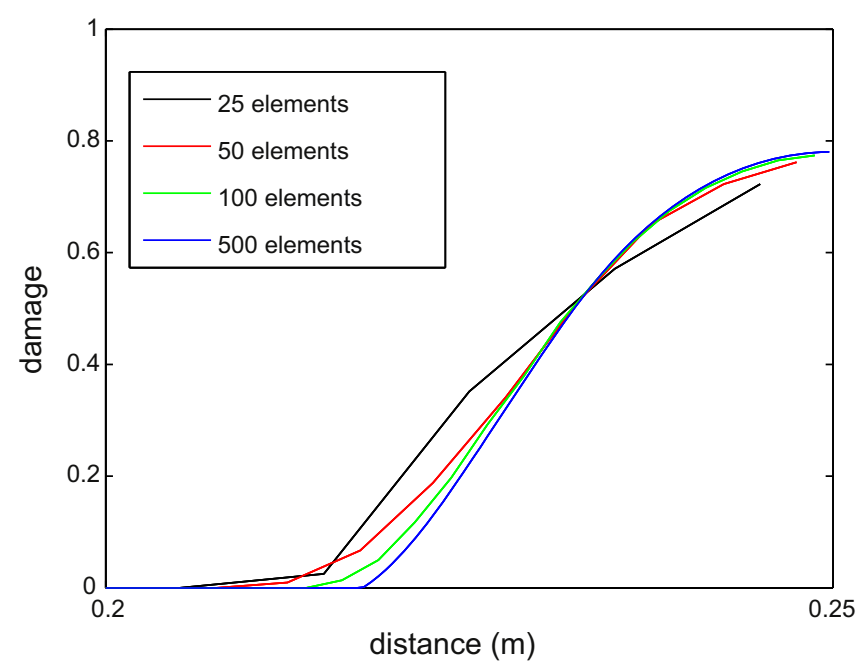

Fig. 4. Mesh objectivity of model.

been run for the same time period reduces the peak damage experienced. Using a (relatively) very small value for the micro structural length scale leads to a brittle response and a damage profile which becomes confined to a single element and thus results in mesh dependent response. The width of the localisation zone as predicted by the dispersion analysis in equation (17), is $0.0721 \mathrm{~m}$ and $0.12 \mathrm{~m}$ for micro structural length scales of $3 \times 10^{-3} \mathrm{~m}$ and $5 \times 10^{-3} \mathrm{~m}$, respectively. Recognising the symmetry invoked in this example, the numerically observed damage profiles show a very good correlation with the analytical prediction. The regularising properties of the model are further demonstrated by varying the number of elements whilst maintaining a constant stiffness length scale $\ell_{S} 3 \times 10^{-3} \mathrm{~m}$. Fig. 4 shows that the fracture process zone clearly converges with mesh refinement. A decrease in accuracy as the spatial resolution approaches the size of the fracture process zone naturally occurs, however, the damage profile is predominantly controlled by the length scale and not the mesh. Using the finest mesh density of 500 elements, the evolution of the macro and micro strain measures is shown in Fig. 5. It is clear that the micro strain measure $\varepsilon^{\mathrm{m}}$ is more oscillatory in nature than the macro strain measure $\varepsilon^{\mathrm{M}}$.

\subsection{Double notched split Hopkinson bar}

A double notched split Hopkinson bar is now simulated to demonstrate the model in a multi dimensional setting. The spec imen, shown in Fig. 6 , is $50 \mathrm{~mm}$ in height and $10 \mathrm{~mm}$ in width and

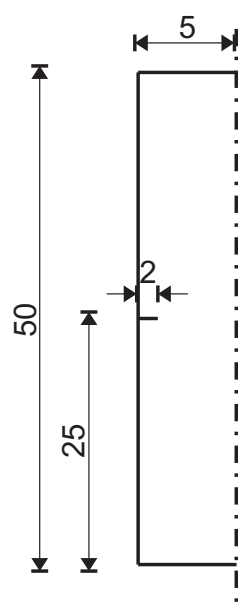

Fig. 6. Double-notched bar geometry.

has a double notch at mid height of $2 \mathrm{~mm}$. Plain strain conditions are assumed and only one half of the specimen is modelled exploiting symmetry. The bar has a Young's modulus of E $30 \times 10^{9} \mathrm{~N} / \mathrm{m}^{2}$, Poisson's ratio $\nu 0.2$ and mass density $\rho 2000 \mathrm{~kg} / \mathrm{m}^{3}$. The micro structural length scale is chosen as $\ell_{\mathrm{s}} \quad 0.5 \times 10^{-3} \mathrm{~m}$. The material degradation of the bar is again modelled using bilinear damage, with a damage initiation strain $\kappa_{\mathrm{i}} \quad 1.2 \times 10^{-4}$ and an ultimate strain of $\kappa_{\mathrm{u}} \quad 1.2 \times 10^{-3}$ as per Fig. 1. The specimen is loaded with a constant pressure of $8 \mathrm{MPa}$ in the negative $y$ direction. For computational convenience the load is actually applied at $20 \mathrm{~mm}$ from the specimen base, thus any wave reflections at the specimen ends have no effect upon the damage accumulated at the notch. The simulations are run for a duration of $5 \mu \mathrm{s}$, with a dilatational wave velocity $c_{p} \quad \sqrt{ }(\lambda+2 \mu) / \rho \quad 4.0825 \times 10^{3} \mathrm{~m} / \mathrm{s}$ (where $\lambda$ and $\mu$ are the Lamé constants), therefore the wave travels a total of $20.4 \mathrm{~mm}$. All results are shown for this final damage state. The specimen is dis cretised using uniform meshes of quadrilateral elements, double nodes have been used at the mid height to create a notch with infinitesimal width. Three densities of meshes have been simulated to check for mesh convergence; mesh 1 uses 1 mm elements, mesh 2 has elements of length $0.5 \mathrm{~mm}$ and mesh 3 of length $0.25 \mathrm{~mm}$. To achieve convergence of the Newton-Raphson iterations, the simulations required 50,100 and 200 times steps for meshes 1,2 and 3 respectively. Fig. 7 shows the contours of damage for the three meshes (with deep red denoting a damage level of 1 and royal blue representing undamaged virgin material), with no appreciable differences in the width of the fracture process zone between the
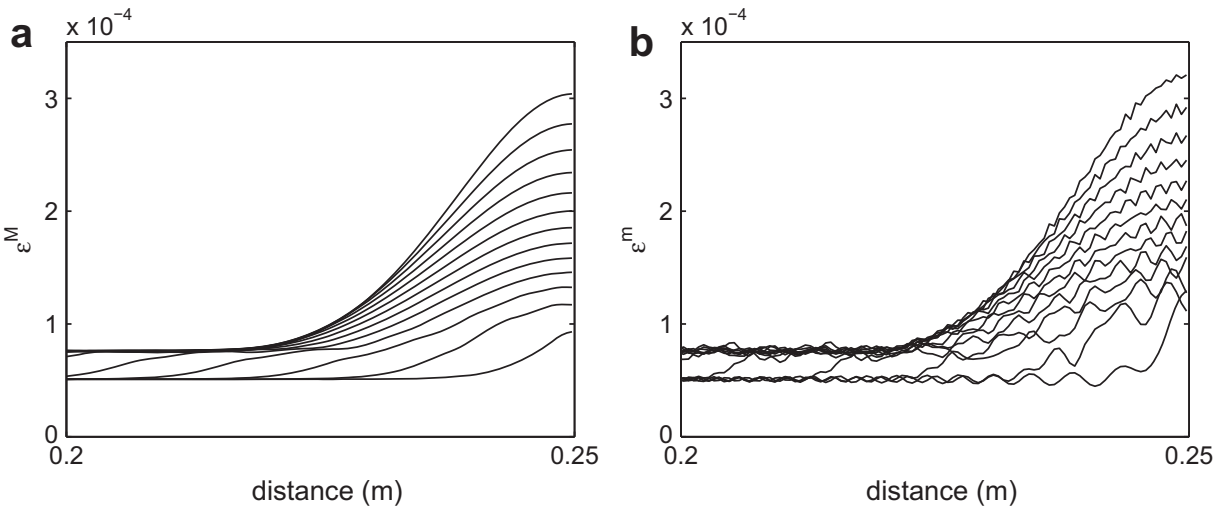

Fig. 5. Evolution of strains a) macro-strains $\varepsilon^{\mathrm{M}}$, b) micro-strains $\varepsilon^{\mathrm{m}}$. 

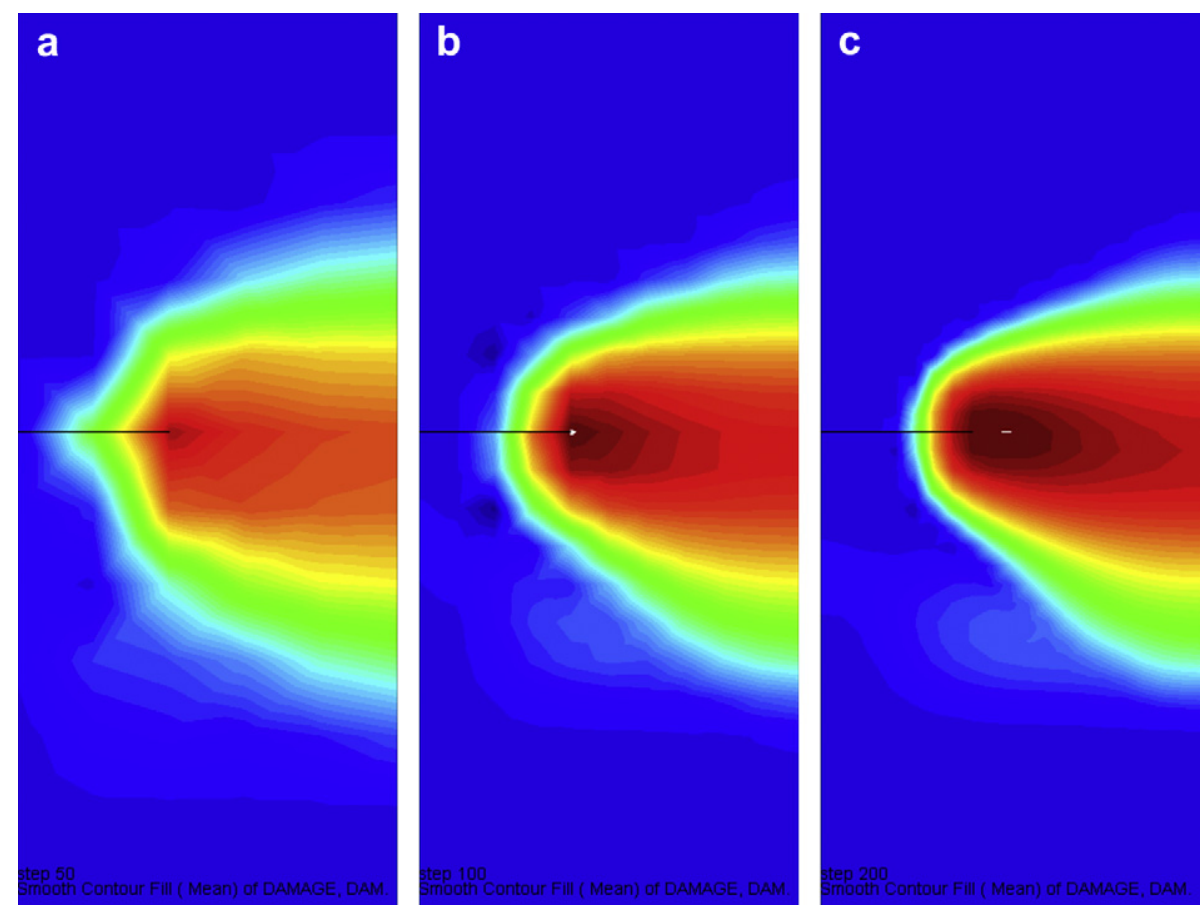

Fig. 7. Damage contours. a) mesh 1, b) mesh 2, c) mesh 3.

two finest meshes. The damage intensity at the tip of the notch does however appear to be influenced slightly by the mesh density. The resulting damage profile is examined in further detail by looking along a line intersecting the notch tip in Fig. 8. The level of damage is plotted against the $y$ coordinate, measured from the base of the specimen, at a distance of $2 \mathrm{~mm}$ in from the edge of the specimen. The reduced level of damage with the $1 \mathrm{~mm}$ mesh is evident along with a slightly wider fracture process zone due to the coarseness of the discretisation. Both the peak damage and size of the process zone are however shown to converge upon mesh refinement.

\subsection{Effects of dispersion on damage}

The one dimensional bar example of Section 3.1 is now revis ited to explore the effect that dispersion has upon damage

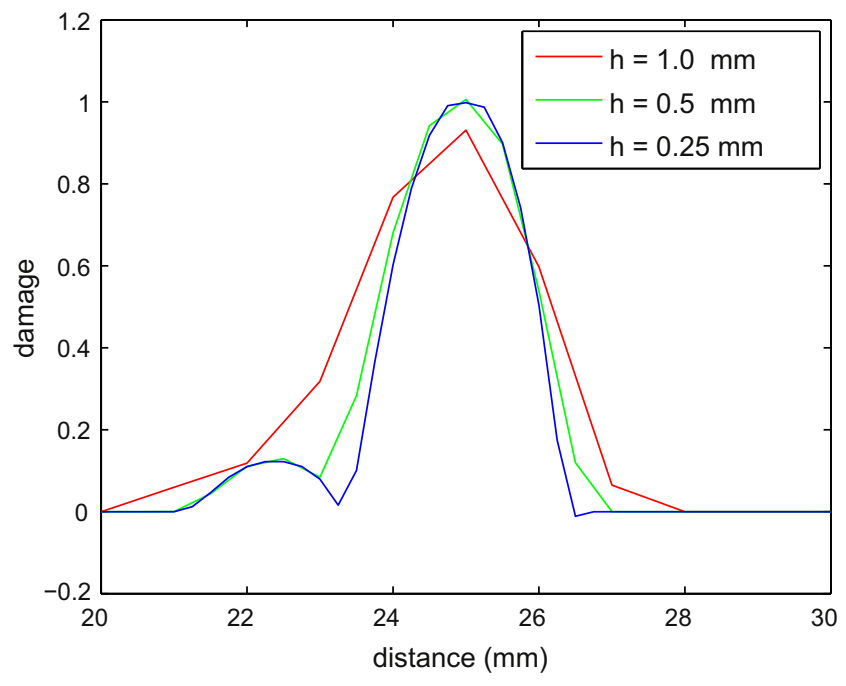

Fig. 8. Damage along line $x \quad 2 \mathrm{~mm}, y \quad 2030 \mathrm{~mm}$. initiation and accumulation. It is instructive when examining fracture initiation to explore first the effects of dispersion upon elastic wave propagation. In Fig. 9, a wavefront created by a Heaviside forcing function is plotted at an instant in time for varying values of the micro inertial length scale $\ell_{m}$ (or more appropriately for this model the ratio of $\ell_{m}$ to $\ell_{s}$ ). The micro structural length scale has been kept constant for all simulations at $\ell_{s} 3 \times 10^{-3} \mathrm{~m}$. The first pertinent feature to note is the effect on the 'local' strain measure $\varepsilon_{\mathrm{m}}$ where, regardless of constitutive model employed for materially non linear behaviour, oscillations about the static strain level will occur due to dispersion and must be considered in any analyses. As can be seen by the $\ell_{m} \quad \ell_{s}$ curve these oscillations can occur as a result of numerical dispersion as well as physical dispersion, in many simulation codes this may not represent a problem due to the use of viscosity or algorithmic damping, for example when using the Hilber-Hughes-Taylor (Hilber et al., 1977) time integration scheme in implicit dynamics. The effects of physical dispersion on damage initiation are best explored by observing the effect that the ratio of $\ell_{\mathrm{m}}$ to $\ell_{\mathrm{s}}$ has upon the wavefront propagation of the 'non local' strain measure $\varepsilon^{\mathrm{M}}$. In Fig. 9b, it can be seen that dispersion has the effect of both extending the toe of the wavefront and altering the peak strain. The effect that this altered wavefront has upon damage accumu lation is now investigated by performing simulations identical to that of Section 3.1 with a variation of the micro inertial length scale $\ell_{\mathrm{m}}$. Fig. 10a shows that for moderate levels of dispersion, damage accumulation is unaffected, confirming the finding of the dispersion analysis in Section 2.2 that the width of the process zone is controlled by the micro structural length scale $\ell_{s}$ alone. For larger levels of dispersion, resulting from a large ratio of $\ell_{m}$ to $\ell_{s}$, the micro inertia length scale $l_{m}$ has an effect on the width of the damage process zone, as shown in Fig. 10b. However, such large levels of dispersion are thought to be physically uncommon. In the case of laminate composites, for instance, it has been shown that large levels of dispersive behaviour could only be encountered if the relative elastic properties of the constituent micro structural materials are dramatically dissimilar (Bennett et al., 2007). 

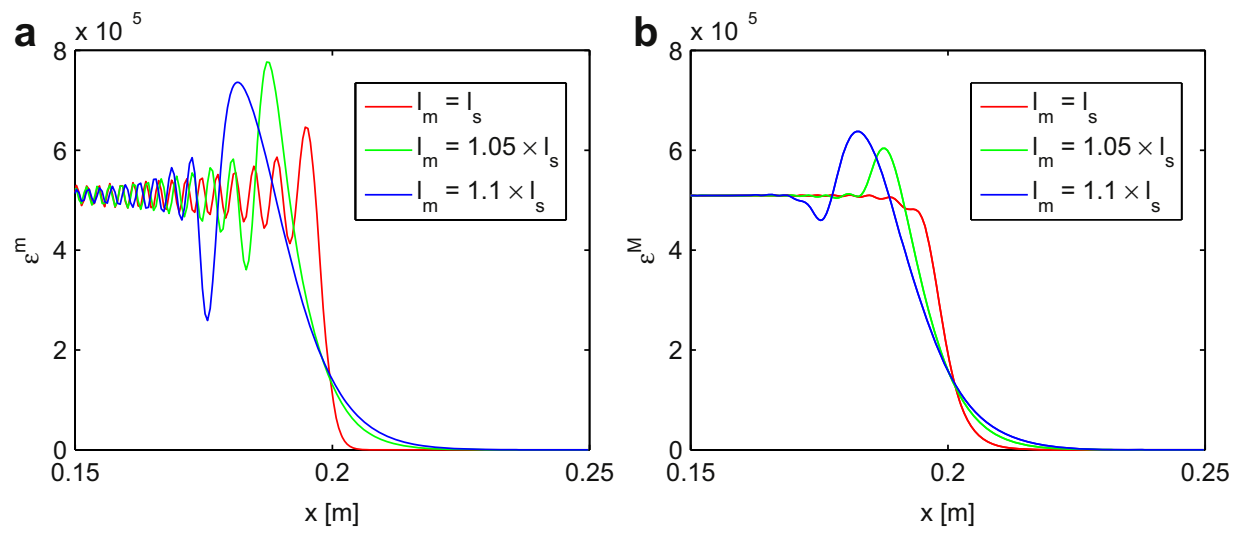

Fig. 9. Dispersive wavefront propagation. a) $\varepsilon^{\mathrm{m}}$, b) $\varepsilon^{\mathrm{M}}$.
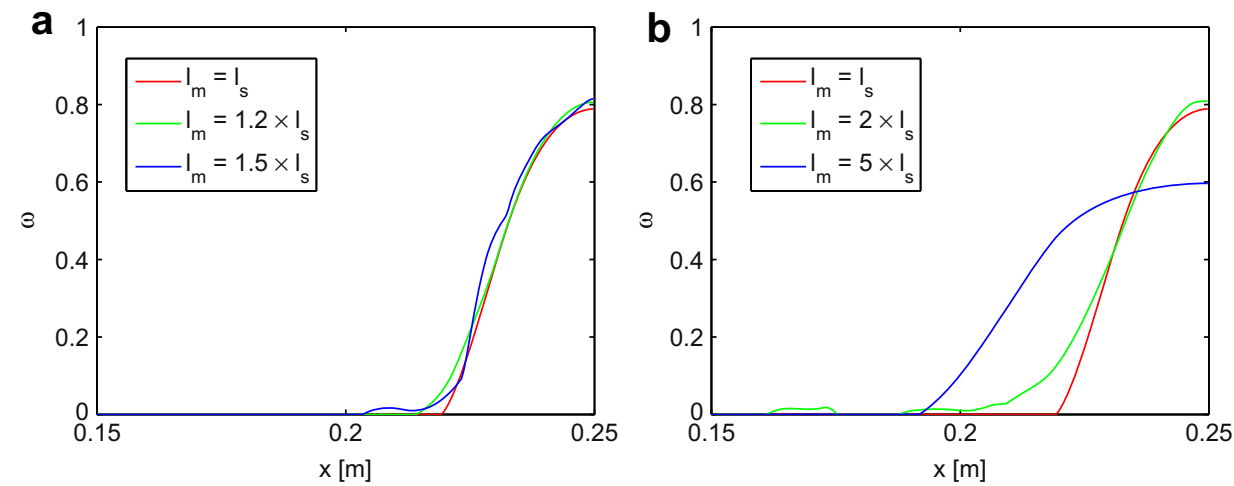

Fig. 10. Influence of dispersion upon damage. a) moderate dispersion, b) large dispersion.

\section{Conclusions}

This paper has demonstrated the use of gradient elasticity as a 'backbone' model to incorporate both regularisation of strain softening and wave dispersion effects into a single model frame work. The 'backbone' nature of the particular implementation of higher order effects means that the constitutive routines are unaffected, thus enabling any constitutive routine to be employed within the framework. Using dispersion analysis it was determined that only one format for a constitutive model can yield a mesh objective model. Whilst this has only been performed here for a continuum damage mechanics model, the results are general. The numerical experiments have confirmed these findings in both one and two dimensional examples. The determination of the critical wave length by means of the dispersion analysis yielded the result that the width of the fracture process zone is dominated by the micro structural length scale parameter. This finding is supported by numerical experiments. The particular implementation of ‘consistent dynamic gradient elasticity' employed has three key features:

- all gradient enhancements can be incorporated into the kinetic energy functional - hence any existing constitutive model can be implemented without interfering with the non local effects.

- a symmetric, positive definite system of equations can be achieved (at least in the elastic range).

- only $\mathscr{C}^{0}$ continuity is required for the spatial discretisation, thus greatly facilitating finite element implementations.

\section{Acknowledgements}

We gratefully acknowledge the financial support of the Engi neering and Physical Sciences Research Council (grants EP/ E01867X/1 and EP/E500544/1) and the Ministerio de Ciencia e Innovación (grants BIA2007 66965 and DPI2007 62395).

\section{References}

Aifantis, E.C., 1992. On the role of gradients in the localization of deformation and fracture. International Journal of Engineering Science 30, 12791299.

Aifantis, E.C., 1999. Strain gradient interpretation of size effects. International Journal of Fracture 95, 299314.

Askes, H., Bennett, T., Aifantis, E.C., 2007. A new formulation and $\mathscr{C}^{0}$-implementation of dynamically consistent gradient elasticity. International Journal for Numerical Methods in Engineering 72, 111126.

Askes, H., Metrikine, A.V., 2002. One-dimensional dynamically consistent gradient elasticity models derived from a discrete microstructure. Part 2: Static and dynamic response. European Journal of Mechanics A Solids 21, 573588.

Bažant, Z.P., Oh, B.H., 1983. Crack band theory for fracture of concrete. Materials and Structures 16, 155177.

Bennett, T., Askes, H., 2008. Finite element modelling of wave dispersion with dynamically consistent gradient elasticity. Computational Mechanics 43, 815825.

Bennett, T., Gitman, I.M., Askes, H., 2007. Elasticity theories with higher-order gradients of inertia and stiffness for the modelling of wave dispersion in laminates. International Journal of Fracture 148, 185193.

Chen, W., Fish, J., 2001. A dispersive model for wave propagation in periodic heterogeneous media based on homogenisation with multiple spatial and temporal scales. Journal of Applied Mechanics Transactions of the ASME 68, 153161.

Georgiadis, H.G., 2003. The mode III crack problem in microstructured solids governed by dipolar gradient elasticity: static and dynamic analysis. Journal of Applied Mechanics Transactions of the ASME 70, 517530 
Georgiadis, H.G., Vardoulakis, I., Lykotrafitis, G., 2000. Torsional surface waves in a gradient-elastic half-space. Wave Motion 31, 333348.

Gitman, I.M., Askes, H., Aifantis, E.C., 2005. The representative volume size in static and dynamic micro macro transitions. International Journal of Fracture 135, L3 L9.

Gutkin, M.Y., Aifantis, E.C., 1999. Dislocations in the theory of gradient elasticity. Scripta Materialia 40, 559566.

Hilber, H.M., Hughes, T.J.R., Taylor, R.L., 1977. Improved numerical dissipation for time integration algorithms in structural dynamics. Earthquake Engineering and Structural Dynamics 5, 283292.

Mazars, J., 1986. A description of micro- and macroscale damage of concrete structures. Engineering Fracture Mechanics 25, 729737.

Metrikine, A.V., Askes, H., 2002. One-dimensional dynamically consistent gradient elasticity models derived from a discrete microstructure. Part 1: Generic formulation. European Journal of Mechanics A/Solids 21, 555572

Metrikine, A.V., Askes, H., 2006. An isotropic dynamically consistent gradient elasticity model derived from a 2D lattice. Philosophical Magazine 86, 32593286.

Mindlin, R.D., 1964. Micro-structure in linear elasticity. Archive for Rational Mechanics and Analysis 16, 5178
Muhlhaus, H.B., Aifantis, E.C., 1991. A variational principle for gradient plasticity. International Journal of Solids and Structures 28, 845857.

Peerlings, R.H.J., de Borst, R., Brekelmans, W.A.M., de Vree, J.H.P., 1996. Gradient enhanced damage for quasi-brittle materials. International Journal for Numerical Methods in Engineering 39, 33913403.

Pijaudier-Cabot, G., Bažant, Z.P., 1987. Nonlocal damage theory. ASCE Journal of Engineering Mechanics 113, 15121533

Rodríguez-Ferran, A., Bennett, T., Askes, H., Tamayo-Mas, E., 2011. A general framework for softening regularisation based on gradient elasticity. International Journal of Solids and Structures 48, 13821394

Ru, C.Q., Aifantis, E.C., 1993. A simple approach to solve boundary-value problems in gradient elasticity. Acta Mechanica 101, 5968.

Sluys, L.J., 1992. Wave propagation, localisation and dispersion in softening solids. Ph.D. thesis, Delft University of Technology.

Toupin, R.A., 1962. Elastic materials with couple-stresses. Archive for Rational Mechanics and Analysis 11, 385414

Wang, Z.P., Sun, C.T., 2002. Modeling micro-inertia in heterogeneous materials under dynamic loading. Wave Motion 36, 473485 